NOTE

\title{
Reliability of VHF telemetry data for measuring attendance patterns of marine predators: a comparison with time depth recorder data
}

\author{
Andrew D. Lowther ${ }^{1, *}$, Heidi Ahonen ${ }^{1}$, Greg Hofmeyr ${ }^{2,3}$, W. Chris Oosthuizen ${ }^{4}$, \\ P. J. Nico De Bruyn ${ }^{4}$, Christian Lydersen ${ }^{1}$, Kit M. Kovacs ${ }^{1}$ \\ ${ }^{1}$ Norwegian Polar Institute, Fram Centre, 9296, Tromsø, Norway \\ ${ }^{2}$ Port Elizabeth Museum at Bayworld, Humewood 6013, Port Elizabeth, South Africa \\ ${ }^{3}$ Department of Zoology, Nelson Mandela Metropolitan University, Summerstrand 6031, Port Elizabeth, South Africa \\ ${ }^{4}$ Mammal Research Institute, Department of Zoology and Entomology, University of Pretoria, Private Bag X20, Hatfield, \\ Pretoria 0028, South Africa
}

\begin{abstract}
Very high frequency (VHF) radiotelemetry data has been used for over $30 \mathrm{yr}$ to monitor the behavior patterns of otariid seals. These data have been used in a wide variety of ways, from characterizing the reproductive and foraging ecology of these species to inferring ecosystem changes based on variation in attendance patterns. Yet the accuracy of VHF data has never been appropriately evaluated. Our study compares VHF data collected on 16 lactating Antarctic fur seals to assess onshore attendance with concurrently collected time depth recorder (TDR) data used as the 'true' measurement of time spent onshore. Within the retrieved datasets, $25 \%$ of the VHF data could not be interpreted with any reliability. Additionally, there were significant differences in the number and duration of attendance bouts between the 2 instrument types, with VHF data overestimating attendance bout duration by approximately $8.9 \mathrm{~h}$ on average. Importantly, the magnitude and direction of errors between VHF and TDR measurements were not systematic, suggesting that VHF data is an inappropriate method for collecting attendance data. Modelling the raw VHF data in a state-space framework elicited mean attendance durations that were indistinguishable from TDR-derived measurements, suggesting this approach may provide a means to re-examine historic VHF data. Moreover, given the evolution of electronic tags in terms of sophistication, miniaturization, longevity and decreasing cost over the last $30 \mathrm{yr}$, TDRs are a more appropriate means of collecting attendance data on centrally foraging marine mammals such as otariids.
\end{abstract}

KEY WORDS: Otariid · Ecosystem monitoring - CCAMLR - Telemetry · Diving · Attendance patterns

Resale or republication not permitted without written consent of the publisher

\section{INTRODUCTION}

Very high frequency (VHF) telemetry has been used to monitor marine mammal movement for over 50 yr (Baldwin 1965), becoming a favored method for characterizing attendance patterns of lactating otariid seals (fur seals and sea lions) during the 1980s (Loughlin et al. 1987), and it is still used in contemporary studies (McDonald et al. 2012, Riet-Sapriza et al. 2012). It has also been the standard method in the Commission for the Conservation of Antarctic Marine Living Resources (CCAMLR) Ecosystem Monitor- 
ing Program (CEMP) for quantifying inter-annual variation in onshore attendance patterns of Antarctic fur seals Arctocephalus gazella and 4 penguin species since the inception of the program in 1989 (CCAMLR 2004). CCAMLR is responsible for managing the harvest of living resources in the Southern Ocean, including krill Euphausia superba (Edwards \& Heap 1981). The krill fishery is the largest by biomass in the Southern Ocean and has the potential to expand over the coming decades (Nicol et al. 2012). CCAMLR has adopted an ecosystem-based approach to managing this fishery and aims to ensure that harvesting is sustainable, not only for the harvest species, but also for krill-dependent predator species such as seals and penguins. Consequently, the measurements used by CCAMLR have an applied outcome, and as such should be scrutinized in terms of how accurately they reflect the biological parameters they are designed to monitor. Verifying the appropriateness of such data is increasingly important given the growing body of evidence supporting inter- and intra-individual variability in reproductive and foraging ecology (Bolnick et al. 2003).

In light of this, our study explicitly tested the accuracy of VHF-derived measurements of onshore attendance used by CEMP on a krill-predating species, the Antarctic fur seal. We used a doubletagging experimental design, simultaneously deploying VHF and TDRs on individual seals. The wet/dry sensors of the TDRs were backed up by diverelated data providing an accurate means of identifying periods when individuals were in the water. We examined VHF attendance data to determine its accuracy compared to dive-instrument data. Additionally, we applied a novel state-space modeling technique to the raw VHF data to determine whether this can improve the accuracy of onshore attendance data from this instrument type.

\section{MATERIALS AND METHODS}

This study was conducted on the subantarctic island of Bouvetøya in the Southern Ocean $\left(54^{\circ} 23^{\prime} \mathrm{S}\right.$, $3^{\circ} 47^{\prime} E_{\text {; Fig. } 1)}$ as part of the 2014-2015 Norwegian Antarctic Research Expedition (NARE). The island hosts the world's second largest breeding population of Antarctic fur seals, which occupies a rocky beach on the northwest corner of the island called Nyrøysa (Fig. 1). This beach has been a CEMP site since 1996. A new research base (Norvegia Station) is situated on the northern slope of Nyrøysa, $83 \mathrm{~m}$ above sea level, giving an unrestricted view of the main breed- ing beach and an ideal platform for reception of VHF signals (Fig. 1).

\section{Instrumentation}

This study was part of a larger CEMP undertaking conducted at Bouvetøya from 12 December 2014 to 11 February 2015. Between 15 and 19 December 2014, 20 lactating adult female Antarctic fur seals observed with suckling pups were captured and manually restrained in a hoop net. Each animal was double-tagged with a VHF transmitter (model V2G 154C, Sirtrack; www.sirtrack.com) and an archival time depth recorder (TDR-Mk9, Wildlife Computers; www.wildlifecomputers.com). Instruments were attached to the fur mid-dorsally in a line starting with the VHF tag at the midpoint between the foreflippers using quick-set epoxy glue (Araldite ${ }^{\circledR}$ 2012). VHFs were set to transmit a signal every $2 \mathrm{~s}$ on unique frequencies at $0.02 \mathrm{MHz}$ increments (range 140 to $150 \mathrm{MHz}$ ), with an estimated detection range and lifespan of $2 \mathrm{~km}$ and $6 \mathrm{mo}$, respectively (Sirtrack). The TDRs recorded data on 3 separate channels related to the different onboard sensors (pressure, wet/dry contacts and light). The pressure channel was set to record data every second, and the wet/dry sensor channel was set to record every $10 \mathrm{~s}$ to avoid filling the device memory prior to recapture. Determining whether the TDR is wet or dry is based on the electrical resistance across the wet/dry contacts; as the contacts dry out, resistance increases. Wet/dry sensor data is recorded as a numeric value reflecting the practical salinity unit (PSU), a measure of seawater conductivity that typically ranges from 35 (complete conductivity, i.e. wet, in seawater) to 255 (complete resistance, i.e. dry, out of water) (Wildlife Computers Mk9 user manual). The threshold at which a 'dry' event is recorded can be set a priori if desired, and the device can be set to stop recording data once this threshold is reached. However, we chose to set the instruments to continuously record the numeric measurement of seawater electrical conductivity so that the record could then be checked for when the animal is in sea water (or not), rather than whether the instrument was wet or dry (based on pre-set values). We set the dive recorders to sample whether they were wet or dry, avoiding the need to set a wet/dry threshold conductivity value, providing us with the full dataset from the instruments and allowing us to not rely on instrumentbased pre-processing. Animals were recaptured using the same technique outlined earlier, approxi- 
mately 5 weeks after instrument deployment, to remove the TDRs.

\section{VHF data recording}

A Telonics TR-5 Scanning Receiver (Telonics www.telonics.com) with an integrated data acquisition system and a RA-5A omni-directional antenna with a $3 \mathrm{dBd}$ gain were used to record VHF data from the instrumented animals between 15 December 2014 and 29 January 2015. Individual frequencies were scanned every $25 \mathrm{~min}$ for $8 \mathrm{~s}$, and if a signal of sufficient quality was received during this period the receiver recorded transmissions for a further $15 \mathrm{~s}$, up to a maximum of 15 pulses (Boyd et al. 1991). We define a signal as having sufficient quality when its pulse width is between 12 and 20 milliseconds (ms) with a pulse interval between 1700 and $2400 \mathrm{~ms}$. All VHF loggers were tested against these parameters prior to deployment. The receiver was run continuously on $240 \mathrm{~V}$ AC power supplied through the research station with a backup battery-pack capable of sustaining the device for up to $24 \mathrm{~h}$. The antenna was mounted with a magnetic base on the roof of the station, providing an unobstructed view of the main breeding beach (Fig. 1). VHF data downloading took less than $1 \mathrm{~h}$ every second day, with the exception of one prolonged download on 21 December that resulted in the receiver being offline for $3.4 \mathrm{~h}$ (see Fig. S1 in the Supplement at www.int-res.com/ articles/suppl/m538p249_supp.pdf). During these offline periods, data were recorded by hand from detections made by a second Telonics TR-5 receiver set to the same specifications and antenna configuration being used in other studies.

(a)


(b)

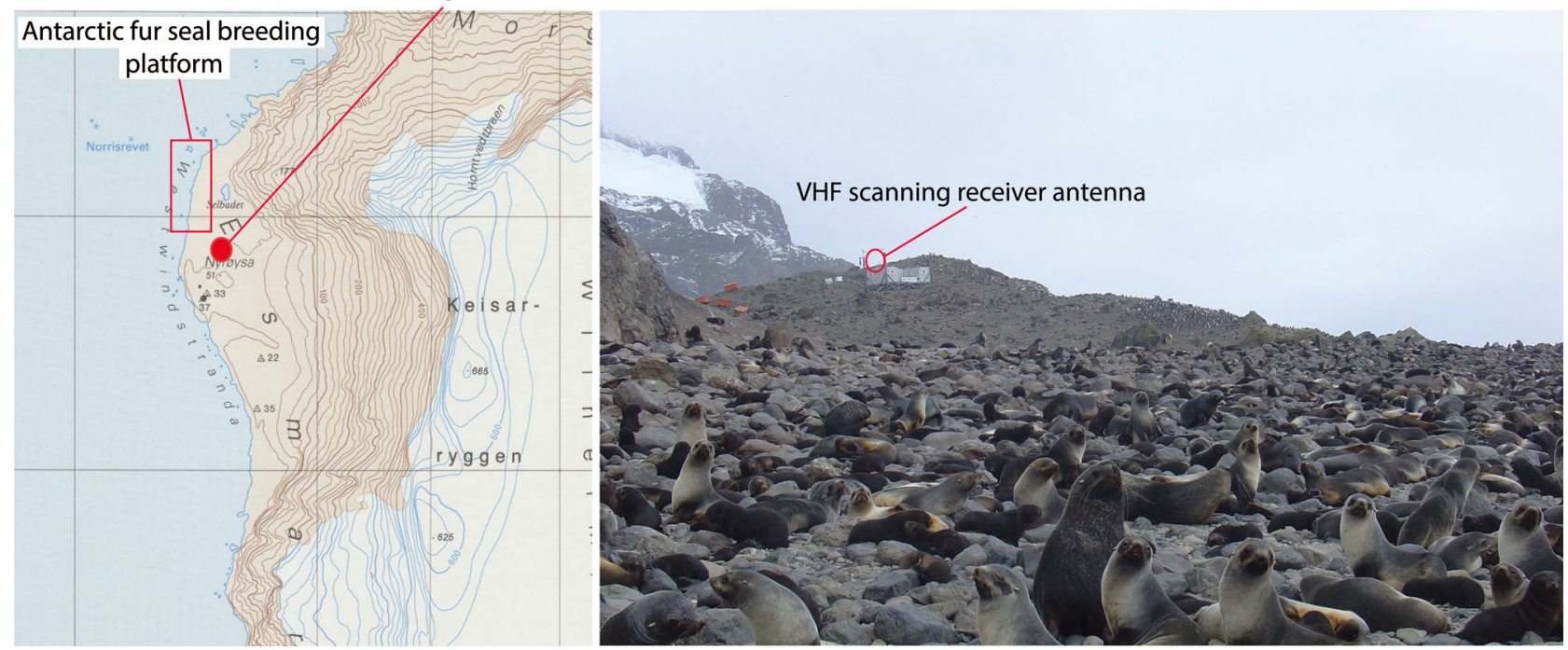

Fig. 1. (a) Bouvetøya, in the southwest Atlantic Ocean, has been a CCAMLR Ecosystem Monitoring Program site since 1997. The main breeding platform is at Nyrøysa on the northwest corner of the island, and hosts over 60000 adult Antarctic fur seals Arctocephalus gazella, which produce between 12000 and 15000 pups each austral summer breeding season. (b) The Norwegian Polar Institute base (Norvegia Station) is situated on high ground, offering an unobstructed view of the main breeding platform where all the animals in this study were instrumented. The VHF scanning receiver was installed in the station, and the antenna mounted on the roof using a magnetic base 


\section{Data processing and analysis}

We used 3 different approaches to characterize the number and duration of onshore attendance patterns of adult female Antarctic fur seals.

(1) Raw VHF data. Downloaded VHF TR-5 files were imported into the $\mathrm{R}$ statistical environment ( $\mathrm{R}$ Core Team 2013) and binned into hourly groups using custom-written functions. Antarctic fur seals typically attend their offspring for at least $12 \mathrm{~h}$, with most attendance bouts being longer than $43 \mathrm{~h}$ (Boyd et al. 1991, Goldsworthy 1999). Consequently, attendance patterns from raw VHF data were derived visually by selecting the first and last hourly bin of data records longer than 12 consecutive hours (see Fig. 2) (Boyd et al. 1991).

(2) Modeled VHF data. We employed a 2-step modeling approach to estimate the probability of an individual being onshore at any given time. Adult female Antarctic fur seals come onshore to nurse their land-based offspring and subsequently return to sea, likely following some endogenous cue; thus, the probability of an individual being onshore through time is unlikely to be geometrically distributed (i.e. the probability of being onshore at time $t$ is dependent on being onshore at time $t-1$ ). Consequently, we made use of the semi-Markov nature of the time-series VHF data to estimate the probability of an individual being in a hidden (unobservable) state, conditional on the amount of time it had already been in that state. Step 1 involved fitting a series of hidden semi-Markov models (HSMM) to the hourly binned VHF data to estimate the time at which individuals switched from the hidden (unobserved; presumably at sea) state to being electronically observed onshore. Models were fitted using the 'hsmm' package in R (Bulla \& Bulla 2013) with a Bernoulli distribution for the conditional observations and a Poissondistributed run length. Model fitting was performed using an iterative expectation-maximization (EM) algorithm which requires an initial set of parameters. Accordingly, run length and conditional observation distributions were initialized with the parameters derived from fitting the relevant distributions to the hourly binned raw data, and a 2-state (onshore versus unobserved) transition probability matrix was initially set to $\mathrm{P}($ onshore $)=0.8$ and $\mathrm{P}($ unobserved $)=0.2$. Step 2 involved estimating the most probable state of an individual at any given time using the fitted models above as input into the hsmm.smooth function in the 'hsmm' package. The most probable time at which an individual transitions between states was then identified and used to define onshore attendance patterns.
(3) Dive recorder data. Data from dive depth and wet/dry sensor channels were initially downloaded from the TDRs using proprietary software (Mk9 Host $v 1.09 .1028)$. Raw data were then imported into the $R$ statistical environment and both channels were plotted against time using the 'diveMove' package (Luque \& Fried 2011). Onshore attendance bouts were identified visually, first using continuously high wet/dry sensor values that were sustained for periods greater than $12 \mathrm{~h}$ and confirmed by an absence of pressure sensor data indicating diving. The time and dates for onshore attendance bouts were extracted and subsequently used to reflect 'true' attendance data.

Differences in the number and duration of attendance bouts recovered using each approach were tested for significance with paired Mann Whitney $U$-tests.

\section{RESULTS}

Of the 20 animals initially instrumented, 19 were successfully recaptured, and at the time of recapture, all instrumented animals still had suckling pups. Equipment failures resulted in only 16 complete datasets being collected. Of the remaining datasets, 4 $(25 \%)$ failed to produce VHF datasets for which onshore attendance bouts of durations $>12 \mathrm{~h}$ could be identified (see Fig. S1 in the Supplement for examples of unworkable data). This resulted in complete datasets from 12 individuals that were available for further analysis. Across all individuals, raw VHF, HSMMmodeled VHF and TDR data generated mean $( \pm \mathrm{SD})$ attendance bout durations of $52 \pm 12.91 \mathrm{~h}$ (range $=$ $30.2-76.3 \mathrm{~h}), 38.9 \pm 15.41 \mathrm{~h}(\mathrm{range}=14.2-77.6 \mathrm{~h})$, and $46.8 \pm 31.77 \mathrm{~h}$ (range $=23.6$ to $143.5 \mathrm{~h}$ ), respectively. The same derived datasets recorded mean attendance bouts of $5.9 \pm 2.7,4.8 \pm 1.1$, and $4.2 \pm 1.1$, respectively, during the experimental period.

Generally, compared to TDR-derived data, raw VHF data significantly overestimated the duration of foraging trips by a mean of $8.9 \mathrm{~h}(95 \% \mathrm{CI}:-6.7$ to $22.3 \mathrm{~h}$; Mann Whitney $U$-test $U=850, \mathrm{p}<0.01$ ), with the exception of 4 individuals where this pattern was reversed (Table 1). This trend was not detected when using HSMM-modeled VHF data (Mann Whitney $U$-test $U=650, \mathrm{p}=0.45$; Table 1, Fig. 2). Similarly, raw VHF data significantly overestimated the number of onshore attendance bouts by at least one in $50 \%$ of the individuals (Mann Whitney $U$-test $U=15, \mathrm{p}=0.03$; Table 1, Fig. 2). Importantly, the magnitude and direction of error between VHF-derived attendance patterns was not systematic between individuals (Fig. 3). 
Table 1. Instrument-derived mean $( \pm \mathrm{SD})$ measurements of onshore attendance for 12 lactating adult female Antarctic fur seals Arctocephalus gazella followed at Bouvetøya during the 2014-2015 austral summer. Very high frequency (VHF) data are presented 'raw' and also as fitted values derived from hidden semi-Markov models (HSMM) to incorporate levels of uncertainty associated with detecting the signals. Time measured by wet/dry and pressure sensors of time and depth recorders (TDR) are assumed to represent the 'truth'. Across all animals, HSMM-modeled VHF data matched most closely with TDR data. The raw VHF data overestimated attendance durations in 8 cases and underestimated them in the remaining 4. HSMMmodeled VHF data tended to identify the number of onshore attendance bouts more correctly than the raw VHF data

\begin{tabular}{|c|c|c|c|c|c|c|}
\hline \multirow[t]{2}{*}{ VHF tag } & \multicolumn{3}{|c|}{ - Duration (h) } & \multirow[b]{2}{*}{ Raw } & \multirow{2}{*}{$\begin{array}{l}\text { Trips (n) } \\
\text { HSMM }\end{array}$} & \multirow[b]{2}{*}{ TDR } \\
\hline & Raw & HSMM & TDR & & & \\
\hline 148.081 & $40.8 \pm 28.16$ & $30.9 \pm 22.04$ & $41.1 \pm 26.89$ & 7 & 7 & 6 \\
\hline 148.121 & $76.3 \pm 29.82$ & $77.6 \pm 32.11$ & $39.1 \pm 31.83$ & 5 & 5 & 5 \\
\hline 148.440 & $52.4 \pm 20.78$ & $36.5 \pm 7.80$ & $35.1 \pm 16.39$ & 3 & 3 & 4 \\
\hline 148.500 & $47.3 \pm 26.02$ & $31.9 \pm 12.67$ & $30.7 \pm 6.63$ & 6 & 5 & 4 \\
\hline 148.600 & $50.5 \pm 31.61$ & $48.1 \pm 31.89$ & $56.7 \pm 62.23$ & 4 & 4 & 4 \\
\hline 148.741 & $54.8 \pm 45.32$ & $29.5 \pm 16.52$ & $29.9 \pm 5.48$ & 7 & 5 & 5 \\
\hline 149.080 & $47.8 \pm 37.17$ & $14.2 \pm 14.31$ & $46.6 \pm 21.57$ & 8 & 6 & 4 \\
\hline 149.401 & $30.2 \pm 5.31$ & $28.3 \pm 12.00$ & $34.0 \pm 18.21$ & 4 & 3 & 4 \\
\hline 149.581 & $73.1 \pm 34.62$ & $38.1 \pm 30.03$ & $143.5 \pm 117.85$ & 8 & 5 & 3 \\
\hline 149.640 & $56.1 \pm 22.63$ & $47.4 \pm 24.02$ & $48.2 \pm 28.98$ & 5 & 5 & 5 \\
\hline 149.719 & $54.3 \pm 37.22$ & $43.8 \pm 32.29$ & $33.7 \pm 1.87$ & 4 & 4 & 2 \\
\hline 149.839 & $40.8 \pm 23.73$ & $41.3 \pm 25.46$ & $23.6 \pm 17.20$ & 3 & 3 & 3 \\
\hline
\end{tabular}

(a)

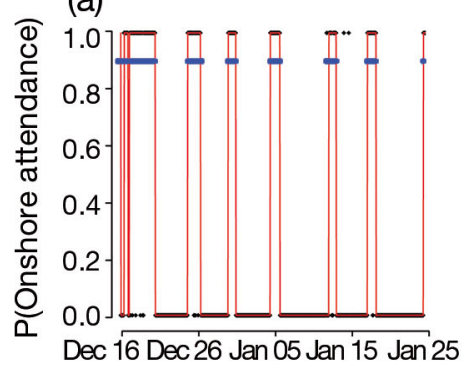

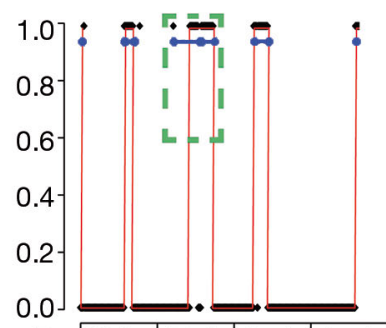

Dec 16 Dec 26 Jan 05 Jan 15 Jan 25 (b)
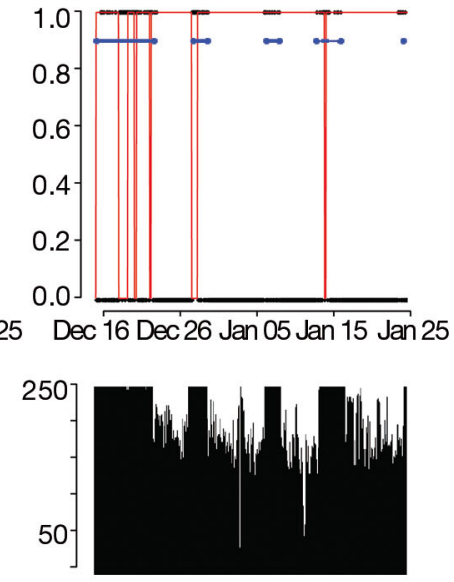

0

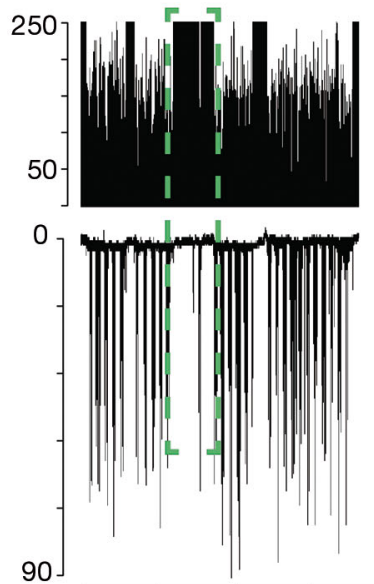

90

(1)

Dec 16 Dec 26 Jan 05 Jan 15 Jan 25

Dec 16 Dec 26 Jan 05 Jan 15 Jan 25 Dec 16 Dec 26 Jan 05 Jan 15 Jan 25
Date

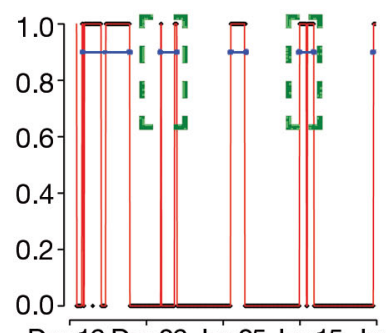

Dec 16 Dec 26 Jan 05 Jan 15 Jan 25



90

Dec 16 Dec 26 Jan 05 Jan 15 Jan 25

Fig. 2. Examples of instrument data from 4 of 12 Antarctic fur seals Arctocephalus gazella dual tagged with very high frequency (VHF) and time depth recorder (TDR) devices at Bouvetøya between December 2014 and January 2015. Top panels of each plot represent VHF data (black dots) binned into hours and fitted with a hidden semi-Markov model to determine the probability of being observed onshore. Red lines: transitions between being detected onshore to presumed at-sea foraging. Interconnected blue dots: onshore attendance periods determined by TDR wet/dry sensor data and offset on the $y$-axis for clearer representation. Bottom panels show wet/dry sensor and dive data for each individual during the instrumentation period. Larger wet/dry sensor values indicate increased resistance between saltwater contacts, which is assumed to indicate that the individual was onshore. Panels (a) and (b) show individuals with the highest and lowest quality VHF data, respectively. Although VHF tags were left attached after the TDRs were removed, data are truncated to the time of recapture for the purpose of this study. Dashed green boxes: anomalies between VHF and TDR-derived measures of onshore attendance 


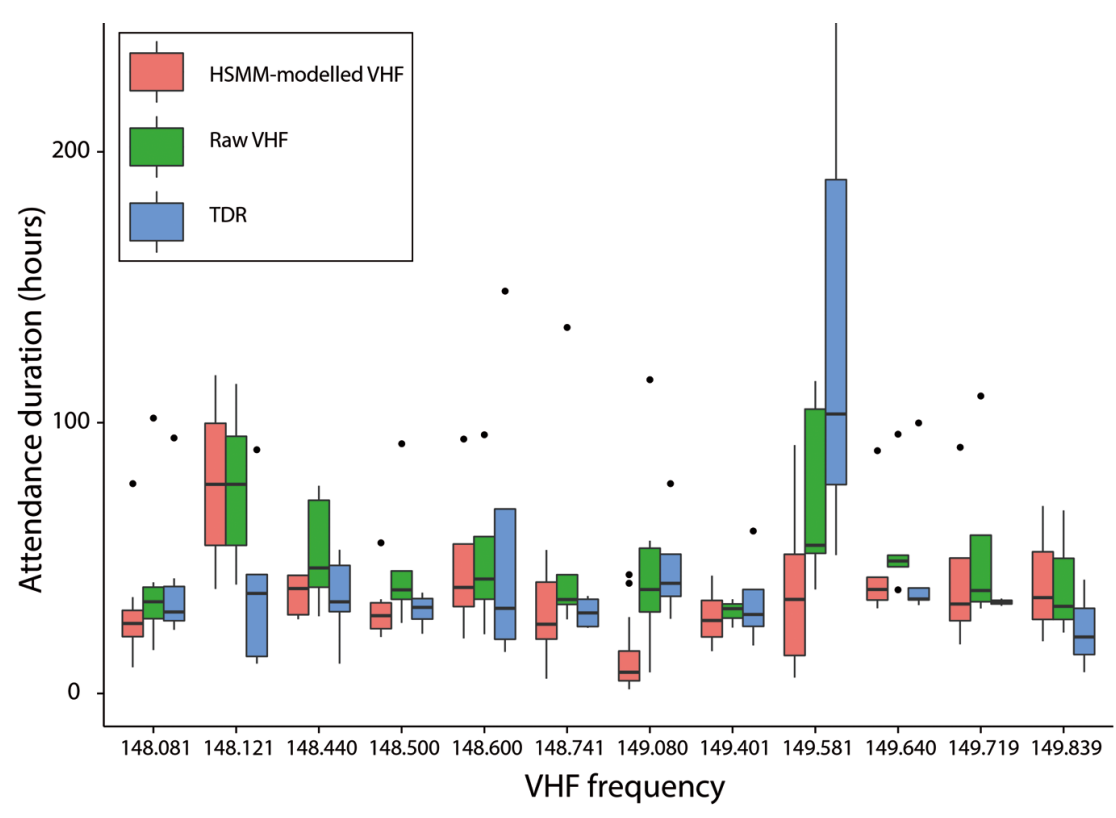

Fig. 3. Very high frequency (VHF; raw and hidden semi-Markov modeled, HSMM) and time depth recorder (TDR) data used to characterize mean onshore attendance patterns from 12 Antarctic fur seals Arctocephalus gazella dual tagged with VHF and TDR devices at Bouvetøya between December 2014 and January 2015. Note the degree of error in VHF-derived attendance data is not consistent between individuals when compared to the true attendance patterns determined using TDR data. •: outlier values. Box and whisker plots represent median values, with the hinges identifying the interquartile range (IQR). Whiskers represent $1.5 \times$ IQR

accurately VHF telemetry data reflects true attendance patterns. We have demonstrated that standard approaches using raw VHF data significantly underestimate or overestimate the number and duration of attendance bouts performed by lactating Antarctic fur seals, and that this overestimation is not consistent across individuals. Modeling the raw VHF data under a hidden semi-Markov framework significantly reduces the levels of inaccuracy when compared to TDR-derived attendance patterns and may be an appropriate method to apply to previously collected radiotelemetry datasets to improve time-trend analyses.

A radio wave emitted from a point source, propagating through an environment free of reflecting or absorbing boundaries, loses intensity at a proportional rate in relation to the inverse square of the distance travelled. Given that (1) the supplier-estimated detection range of the transmitters used in our study was $>2 \mathrm{~km}$, while the

\section{DISCUSSION}

Behavioral studies that involve double-tagging fur seals with VHF and TDR instruments such as ours have been conducted since the early 1990s (e.g. Boyd et al. 1991). However, the earlier studies utilized the devices for separate purposes, relying on VHF telemetry data to infer that an animal was onshore and TDR data to characterize its at-sea foraging behavior (Boyd et al. 1994, Francis et al. 1998, Georges \& Guinet 2000). Importantly, in terms of applied outputs such as fisheries management, VHF telemetry data has been used in studies to characterize geographical variation and interannual changes in the duration and number of onshore attendance bouts of krill predators such as fur seals (Reid 2002) and penguins (Jansen et al. 2002) within the CEMP monitoring program. In turn, changes in these metrics are interpreted as changes in components of the ecosystem, namely changes in the abundance and temporal or spatial distribution of krill (Agnew 1997). Central to this rationale is an accurate and reproducible means to assess the behavioral patterns of animals. To our knowledge, our study is the first to look at how receiver antenna was mounted $<500 \mathrm{~m}$ from the breeding area in direct line-of-sight, and (2) all the individuals (whose instruments functioned) were detected by VHF to some degree throughout the study period, we believe our experimental design is robust enough to support our conclusions. Thus, the asymmetrical variability in detection we describe is most likely due to individual differences in the haulout behavior of adult females, in terms of where they choose to nurse their pups and what body position they adopt while nursing, both of which can lead to obstructions in VHF transmission. Such behaviors are likely to be impacted by local topography and therefore be unpredictable across populations and landscapes, meaning that simply providing a 'correction factor' to the VHF data will not result in improved data quality. There are a considerable number of challenges to overcome and subjective assumptions to be made when collecting and analyzing VHF data. We thus strongly urge users to consider factors such as geography, harmonic overlap from other transmitters ('signal bleedover'), antenna gain adjustment and frequency bracketing, as well as the interval between successive detection at- 
tempts when designing studies relying solely on VHF telemetry, particularly when cross-site or cross-study comparisons are to be made. For example, our study was part of a larger CEMP program involving over 120 individuals; thus, our selection of detection period was a compromise between maximizing the time to detect each individual and minimizing the amount of time between successive detection events. It is likely that a longer detection period will provide a greater capacity to increase the accuracy and precision of detecting individuals when they are onshore.

Since the inception of VHF telemetry as a technique to measure attendance patterns of fur seals, the price of other more sophisticated electronic logging devices has decreased considerably, while the longevity of data collection times have improved. For example, the Wildlife Computers TDR-Mk9 dive recorders used in this study cost approximately 8 times as much as the Sirtrack VHF tags used herein, yet the battery life of the former devices are capable of sustained usage for 8 times as long as the VHF tags, making the collection of attendance data using TDR instruments cost-neutral, assuming the TDRs can be recovered. Given the relatively restricted movement capabilities of dependent pups, our TDR recovery success rate of approximately $95 \%$ should be considered representative of what can be achieved. However, the risks of relying exclusively on archival loggers, which if not recovered result in a complete loss of data, should not be ignored. Similarly, for long-term studies, the use of more accurate technology should be considered to allow for appropriate cross-study comparisons. Adaptive monitoring regimes can capture older data records during the transition to minimize data loss and maximize a smooth transition to newer technology (Lindenmayer \& Likens 2009). For shorter-term or one-off studies which are subject to financial limitations, VHF technology might remain the only available tool. This is still workable, given the recent advances in digital VHF technology that reduce the impact of several confounding factors introduced by older analogue VHF transmitters. However, we do recommend that long-term research programs that require measurements of onshore attendance collect such data using TDRs or other electronic logging devices, given the unreliability of VHF data, and where this is not feasible, the use of a post-hoc, objective filtering method such as the one we present here should be utilized whenever only VHF data is available. The use of TDRs also provides useful information on the vertical use of space in the water column, which might be important when trying to identify shifts in ecosystem performance such as is attempted by the CCAMLR monitoring system. However, given that VHF data on marine mammal attendance rates have been recorded for decades, modelling techniques such as the one we describe herein can reduce the errors present and make older monitoring data useful for comparative purposes with contemporary, TDRderived datasets.

Acknowledgements. This study was funded by the Norwegian Antarctic Research Expedition (NARE) program awarded by the Norwegian Research Council. We thank the South African National Antarctic Program (SANAP) for providing logistical support in reaching Bouvet. We thank Rudi Menkveld for his help in the field and culinary support. Finally, we thank the 3 anonymous reviewers whose incorporated suggestions improved the manuscript.

\section{LITERATURE CITED}

Agnew DJ (1997) Review - the CCAMLR Ecosystem Monitoring Programme. Antarct Sci 9:235-242

Baldwin HA (1965) Marine biotelemetry. Bioscience 15: 95-97

Bolnick DI, Svanback R, Fordyce JA, Yang LH, Davis JM, Hulsey CD, Forister ML (2003) The ecology of individuals: incidence and implications of individual specialization. Am Nat 161:1-28

Boyd IL, Lunn NJ, Barton T (1991) Time budgets and foraging characteristics of lactating Antarctic fur seals. J Anim Ecol 60:577-592

Boyd IL, Arnould JPY, Barton T, Croxall JP (1994) Foraging behaviour of Antarctic fur seals during periods of contrasting prey abundance. J Anim Ecol 63:703-713

Bulla J, Bulla I (2013) hsmm: Hidden semi-Markov models. $\mathrm{R}$ package version 04, http://cran.r-project.org

CCAMLR (Commission for the Conservation of Antarctic Marine Living Resources) (2004) Standard methods for monitoring parameters of predator species. CCAMLR North Hobart. www.ccamlr.org/ru/system/files/std-meth 04_2.pdf

Edwards DM, Heap JA (1981) Convention on the Conservation of Antarctic Marine Living Resources: a commentary. Polar Rec 20:353-362

Francis J, Boness D, Ochoa-Acuna H (1998) A protracted foraging and attendance cycle in female Juan Fernandez fur seals. Mar Mamm Sci 14:552-574

> Georges JY, Guinet C (2000) Maternal care in the subantarctic fur seals on Amsterdam Island. Ecology 81:295-308

> Goldsworthy SD (1999) Maternal attendance behaviour of sympatrically breeding Antarctic and subantarctic fur seals, Arctocephalus spp., at Macquarie Island. Polar Biol 21:316-325

Jansen JK, Russell RW, Meyer WR (2002) Seasonal shifts in the provisioning behavior of chinstrap penguins, Pygoscelis antarctica. Oecologia 131:306-318

Lindenmayer DB, Likens GE (2009) Adaptive monitoring: a new paradigm for long-term research and monitoring. Trends Ecol Evol 24:482-486

> Loughlin TR, Bengtson JL, Merrick RL (1987) Characteris- 
tics of feeding trips of female northern fur seals. Can J Zool 65:2079-2084

Luque SP, Fried R (2011) Recursive filtering for zero offset correction of diving depth time series with GNU R package diveMove. PLoS One 6:e15850

McDonald BI, Goebel ME, Crocker DE, Costa DP (2012) Biological and environmental drivers of energy allocation in a dependent mammal, the Antarctic fur seal pup. Physiol Biochem Zool 85:134-147

Nicol S, Foster J, Kawaguchi S (2012) The fishery for Antarc-

Editorial responsibility: Peter Corkeron,

Woods Hole, Massachusetts, USA tic krill — recent developments. Fish Fish 13:30-40

R Core Team (2013) R: A language and environment for statistical computing. $\mathrm{R}$ Foundation for Statistical Computing, Vienna. www.R-project.org/

Reid K (2002) Growth rates of Antarctic fur seals as indices of environmental conditions. Mar Mamm Sci 18:469-482

Riet-Sapriza FG, Duignan PJ, Chilvers BL, Wilkinson IS and others (2012) Interannual and individual variation in milk composition of New Zealand sea lions (Phocarctos hookeri). J Mammal 93:1006-1016

Submitted: June 22, 2015; Accepted: September 29, 2015 Proofs received from author(s): October 21, 2015 